\title{
Gambia è (prevalentemente) maschile e gambiani sono i suoi abitanti
}

Paolo D'Achille

PUBBLICATO: 05 APRILE 2019

\section{Quesito:}

Sono arrivati vari quesiti sul genere di Gambia (lo Stato africano, non l'omonimo fuume) e sul relativo etnico: si dice gambiano o gambiese?

\section{Gambia è (prevalentemente) maschile e gambiani sono i suoi abitanti}

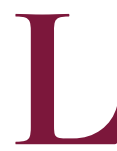

'italiano non ha una regola precisa nell'assegnazione del genere grammaticale ai nomi di regioni e di stati (sulla questione il saggio di riferimento è quello di Anna M. Thornton, L'assegnazione del genere in italiano, in Actas del XXIII Congreso Internacional de Lingüística y Filología Románica, a cura di Fernando Sánchez Miret, vol. I, Tübingen, Niemeyer, 2003, pp. 467-48I): alcuni sono maschili (e si possono appoggiare a un nome comune come appunto Stato, Paese, ma anche regno), altri femminili (e potrebbero appoggiarsi a nomi comuni come nazione, regione, terra, colonia, ma anche repubblica). In genere la terminazione in - a favorisce la scelta del femminile: Austria, Spagna, Russia, Colombia, Guinea, Etiopia, Somalia, Eritrea; si tenga anche presente che alcuni di questi nomi derivano o ricalcano i corrispondenti classici e in latino e in greco sono femminili i coronimi dalla terminazione $-i a$, con $i$ atona, alla latina, o tonica, alla greca. Non mancano tuttavia casi di attribuzione del genere maschile: Canada, Ghana, Kenya. Inoltre, essendo il maschile il genere non marcato, si sono avuti, nel corso del tempo, spostamenti in questa direzione, anche a dispetto della relativa trasparenza del toponimo: è ormai maschile il Venezuela (lett. 'piccola Venezia'), mentre si oscilla tuttora tra la e il Costarica (lett. 'costa ricca').

Nel caso di Gambia, possiamo anzitutto segnalare, sulla base del Deonomasticon Italicum (DI) di Wolfgang Schweickard, vol. II, Tübingen, Niemeyer, 2006, pp. 217-2I8, che il toponimo è documentato in italiano già nel sec. XV come (regno di) Gambra, poi nel XVI come (regno) di Gambea e infine dal I8ı2 come Gambia e che lo Stato prende nome dal fiume che lo attraversa.

Il genere grammaticale, tanto per l'idronimo quanto per il coronimo, ha a lungo oscillato. Nelle prime attestazioni settecentesche reperibili in Google Libri sono entrambi femminili: il fiume in un testo del I738 (la terza ed. del Metodo per istudiare la geografia, in cui si dà un'esatta descrizione dell'universo, estratta da migliori autori, e formata sulle osservazioni dei signori dell'Accademia Reale delle Scienze, con un discorso preliminare su lo studio di questa scienza, ... opera del signor Martineau du Plessis, ritoccata, e accresciuta dal signor Langlet di Fresnoy, vol. I, Napoli, Parrino, p. 226), lo Stato nel titolo del volume nella Nuova geografia di Ant. Federico Busching [...] tradotta in lingua toscana dall'ab. Gaudioso Jagemann, Tomo vigesimo nono, che comprende l'introduzione all'Africa, la Barbaria marittima, e mediterranea, cioè le reggenze di Tripoli, Tunisi, Algeri, l'Impero di Marocco, il Senegal, e la Gambia, la Nigrizia, e la Guinea, Venezia, Zatta, I780 (si noti che questo esempio consente di retrodatare il DI di oltre un ventennio)[qui e oltre neretto nostro].

Ma il nome del fiume è documentato anche al maschile già nel vol. 53 della Parte moderna, ossia Continuazione della Storia universale dal principio del mondo sino al presente scritta da una Compagnia di 
Letterati Inglesi; ricavata da' fonti originali, ed illustrata con Carte Geografiche, Rami, Note, Tavole Cronologiche ed altre; tradotta dall'Inglese, con giunta di Note, e di avvertimenti in alcuni luoghi, Amsterdam [ma Venezia], Paglierini, I788). È questa, del resto, una tendenza generale degli idronimi, che ha portato la Volga e la Piave a diventare il Volga e il Piave (si veda l'articolo di Massimo Fanfani, Fiumi femminili, fumi maschili). Nel sec. XIX le attestazioni al maschile sono numerose, ma esempi al femminile resistono ancora almeno fino al I880 (Nuova enciclopedia italiana, Torino, Utet, p. I264).

Probabilmente il mutamento di genere del fiume ha favorito l'analogo cambiamento di genere del nome dello Stato, che si trova documentato al maschile sul periodico "Il Carabiniere. Giornale militare" del ı887: "I'Inghilterra possiede il Gambia con capitale Bathurst" (p. 151). Il genere dello Stato per tutto il Novecento ha continuato a oscillare tra maschile e femminile, anche se il maschile ha finito col prevalere, come mostrano i dati di Google, relativi sia ai testi a stampa sia alla rete. Il DI segnala la locuzione razza del Gambia come razza di gatto nell'Enciclopedia Italiana Treccani (I932), ma anche la febbre di Gambia 'periodo iniziale o febbrile della malattia del sonno', nel Dizionario Enciclopedico Italiano, sempre della Treccani (1956), in cui l'uso della preposizione semplice evita la scelta del genere grammaticale.

L'assegnazione del femminile non è mai sparita del tutto, grazie alla terminazione in -a (anzi, in -ia) e in alcuni casi potrebbe dipendere anche, come notano alcuni lettori, dalla volontà di differenziare il nome dello Stato (che è oggi una repubblica) da quello del fiume, dove il maschile si è invece stabilizzato. Quanto al genere della nazionale di calcio del Paese, normalmente segue quello dello Stato e in questo caso la prevalenza del maschile pare ancora più netta.

Poiché il nome del Gambia non ricorre molto spesso sulla stampa (neppure su quella sportiva), loscillazione, dovuta a due opposte tendenze tuttora attive nella nostra lingua, sembra destinata a perdurare e la prevalenza del maschile non può ancora essere considerata definitiva; va detto però che nell'archivio della "Repubblica" il Gambia ha 78 occorrenze e la Gambia nessuna.

Quanto all'etnico, il DI registra solo gambiano, attestato come aggettivo nel Vocabolario patronimico italiano o sia Adjettivario italiano di nazionalitá, opera postuma di Francesco Cherubini edita a cura di G.B. De Capitani (Milano, Società Tipografica de' Classici Italiani, I860), e registrato, anche come sostantivo, sia nel GRADIT, sia nel Vocabolario Treccani in rete. Mentre però il primo definisce il lemma come "del Gambia", "nativo o abitante del Gambia", il secondo offre questa definizione, che documenta la possibilità di assegnare allo Stato il genere femminile, che anzi è quello indicato per primo (una scelta che, per le ragioni dette sopra, pare alquanto discutibile):

gambiano agg. e s. m. (f. -a). - Appartenente o relativo alla Repubblica della (o del) Gambia, stato dell'Africa occid. (cosi denominato dal fiume Gambia che l'attraversa); come sost., abitante o nativo della Gambia.

È documentato però anche gambiese, di cui anzi esiste un'attestazione anteriore al ı860: "Ma di lui e dal segretario gambiese non si ebbero altre novelle" (Girolamo Serra, La storia della antica Liguria e di Genova, Capolago, Tipografia Elvetica, vol. IV, I835, p. 276). Questo derivato è tuttora usato, come mostra l'esempio seguente, tratto dall'edizione in rete della "Gazzetta del Mezzogiorno" del ig maggio 2018: "Un cittadino gambiese di 22 anni, Omar Njie, è stato arrestato dalla Polizia a Foggia per rapina e resistenza a pubblico ufficiale". Evidentemente la maggiore produttività del suffisso -ese rispetto ad ano per formare gli etnici (cfr. Franz Rainer, Etnici, in Grossmann-Rainer 2004, pp. 402-408) si fa sentire anche in questo caso. 
In rete si trovano anche attestazioni di gambese, senza la -i, indebitamente considerata elemento desinenziale di Gambia, con particolare riferimento alla valuta locale (la sterlina gambese in passato, il dalasi gambese attualmente). E questo sebbene in inglese si parli di Gambian pound e di Gambian dalasi e anche il francese e lo spagnolo usino rispettivamente gambien e gambiano (si veda, al riguardo, la voce Dalasi nelle varie versioni di Wikipedia).

Isolate attestazioni ha anche gambiense, variante culta di gambiese, che si appoggia al gambiensis/-e del latino scientifico (il protozoo detto Trypanosoma brucei gambiense, che causa la malattia del sonno), e che ha anche qualche isolato esempio in rete fuori da quest'ambito ("Nelle classi in cui collaboriamo, [...] vi è un grande numero di ragazzini provenienti da famiglie marocchine, algerine, gambiensi, rumene e gitane"; Se il servizio civile si trasforma in un abbraccio di un bambino, www.salesianiperilsociale.it/, 28/II/2016). Da notare che in un numero della "Clinica medica italiana" (XLIX, I9IO, p. 631) si parla non di tripanosoma gambiense ma di tripanosoma gambiese.

Insomma, come avviene spesso con gli etnici, specie quelli esotici (si vedano qui le risposte ad analoghi quesiti), le alternative possibili sono molte, ma l'opzione per gambiano sembra proprio quella preferibile.

\section{Cita come:}

Paolo D'Achille, Gambia è (prevalentemente) maschile e gambiani sono i suoi abitanti , "Italiano digitale", 2019, IX, 2019/2 (aprile-giugno)

DOI: $10.35948 / 2532-9006 / 2020.3105$

\section{Copyright 2019 Accademia della Crusca}

Pubblicato con licenza creative commons CC BY-NC-ND 